\title{
Avaliação citomorfométrica de micronúcleos de células epiteliais orais de pacientes
}

\section{fumantes e não fumantes}

\author{
Cytomorphometric evaluation of micronuclei in oral epithelial cells of smoker and non-smoker \\ patients
}

Evaluación citomorfométrica de micronúcleos en células epiteliales orales de pacientes fumadores y no fumadores

Haydee Gina Quispe Jiménez ORCID: https://orcid.org/0000-0002-9622-410X Universidade Federal do Ceará, Brasil E-mail: haydee_qj@hotmail.com Rômulo Melo Mesquita ORCID: https://orcid.org/0000-0002-8475-4840 Universidade Federal do Ceará, Brasil E-mail: romulomelom@gmail.com

Victor Bento Oliveira

ORCID: https://orcid.org/0000-0002-5812-5645 Universidade Federal do Ceará, Brasil E-mail: victorbento@alu.ufc.br

Ana Larissa de Queiroz França ORCID: https://orcid.org/0000-0003-0305-5464 Universidade Federal do Ceará, Brasil

E-mail: larissa_odontoufc@yahoo.com.br

Marcelo Miranda de Melo

ORCID: https://orcid.org/0000-0003-2605-1319 Universidade Federal do Ceará, Brasil

E-mail: marcelomirandamelo3m@gmail.com

Ana Liza Paz Sousa Batista

ORCID: https://orcid.org/0000-0002-0348-4026 Universidade Federal do Ceará, Brasil E-mail: analiza@ sobral.ufc.br

Igor Iuco Castro-Silva

ORCID: https://orcid.org/0000-0003-4815-6357 Universidade Federal do Ceará, Brasil

E-mail: igor.iuco@sobral.ufc.br

\begin{abstract}
Resumo
O rastreamento por citologia esfoliativa de genotoxicidade é uma estratégia importante para mitigação da carcinogênese bucal induzida pelo tabaco. O objetivo deste estudo foi determinar a frequência de micronúcleos em células epiteliais orais de pacientes fumantes e não fumantes. Dezesseis voluntários, de ambos os sexos, 40-60 anos, com mucosa bucal saudável, foram divididos em grupo fumante ou não fumante. Esfregaços citológicos de mucosa jugal, borda lateral da língua e assoalho da boca corados por Papanicolaou permitiram avaliação de 4800 imagens microscópicas. Frequências de células micronucleadas (CMN) e micronúcleos/célula (MN/C) foram comparadas por grupo, região anatômica, sexo e idade, usando o teste two-way ANOVA seguido por Tukey $(p<0,05)$. Houve maior presença de CMN no grupo fumante do que não fumante, com diferenças significativas para assoalho de boca, sexo feminino e faixa de 50-60 anos. A quantidade de 1 ou $2 \mathrm{MN} / \mathrm{C}$ foi maior que 3 ou $\geq 4 \mathrm{MN} / \mathrm{C}$, em especial no grupo fumante. Houve diferenças na frequência de $\mathrm{MN} / \mathrm{C}$ entre fumantes e não fumantes, para as três regiões bucais, para ambos os sexos, embora de forma mais acentuada em mulheres, e para a faixa de 50-60 anos, além da faixa de 40-50 anos para o grupo não fumante. Diferenças de CMN e MN/C segundo regiões bucais, sexo e idade entre fumantes e não fumantes contribuem para compreender a genotoxicidade mediada pelo tabaco.
\end{abstract}

Palavras-chave: Micronúcleos com defeito cromossômico; Patologia; Mucosa bucal; Tabagismo.

\section{Abstract}

Exfoliative cytology screening for genotoxicity is an important strategy for mitigating tobacco-induced carcinogenesis. The aim of this study was to determine the frequency of micronuclei in oral epithelial cells of smoking and non-smoking patients. Sixteen volunteers, of both sexes, 40-60 years old, with healthy oral mucosa were divided 
into smoking or non-smoking group. Cytological smears of buccal mucosa, lateral border of the tongue and floor of the mouth stained by Pap stain allowed evaluation of 4800 microscopic images. Frequencies of micronucleated cells (MNC) and micronuclei/cell (MN/C) were compared by group, anatomical region, sex and age range, using the twoway ANOVA test followed by Tukey $(\mathrm{p}<0.05)$. There was a greater presence of MNC in the smoker than in the nonsmoker group, with significant differences for floor of mouth, female gender and age range of 50-60 years. The amount of 1 or $2 \mathrm{MN} / \mathrm{C}$ was greater than 3 or $\geq 4 \mathrm{MN} / \mathrm{C}$, especially in the smoking group. There were differences in the frequency of MN/C between smokers and non-smokers, for the three oral regions, for both sexes although more accentuated in women, and for the age range of 50-60 years beyond the age range of 40-50 years for the non-smoking group. Differences in MNC and MN/C according to oral regions, sex and age between smokers and non-smokers contribute to understand the genotoxicity mediated by tobacco.

Keywords: Micronuclei chromossome-defective; Pathology; Oral mucosa; Tobacco use disorder.

\section{Resumen}

El cribado mediante citología exfoliativa para la genotoxicidad es una estrategia importante para mitigar la carcinogénesis oral inducida por el tabaco. El objetivo de este estudio fue determinar la frecuencia de micronúcleos en las células epiteliales orales de pacientes fumadores y no fumadores. Dieciséis voluntarios, de ambos sexos, de 40 a 60 años, con mucosa oral sana, se dividieron en grupos de fumadores y no fumadores. Los frotis citológicos de la mucosa de la mejilla, el borde lateral de la lengua y el piso de la boca teñidos por Papanicolaou permitieron la evaluación de 4800 imágenes microscópicas. Se compararon las frecuencias de células micronucleadas (CMN) y micronúcleos/célula (MN/C) por grupo, región anatómica, sexo y edad, utilizando la prueba ANOVA bidireccional seguida de Tukey $(\mathrm{p}<0,05)$. Hubo mayor presencia de $\mathrm{CMN}$ en el grupo fumador que en el grupo no fumador, con diferencias significativas para piso de boca, sexo femenino y rango de 50-60 años. La cantidad de 1 o 2 MN/C fue mayor que $3 \mathrm{o} \geq 4 \mathrm{MN} / \mathrm{C}$, especialmente en el grupo de fumadores. Hubo diferencias en la frecuencia de $\mathrm{MN} / \mathrm{C}$ entre fumadores y no fumadores, para las tres regiones bucales, para ambos sexos, aunque más acentuadas en las mujeres, y para el rango de 50-60 años, además del rango de 40-50 años para el grupo de no fumadores. Las diferencias en CMN y MN/C según regiones orales, sexo y edad entre fumadores y no fumadores contribuyen a comprender la genotoxicidad mediada por el tabaco.

Palabras clave: Micronúcleos con defecto cromosómico; Patología; Mucosa bucal; Tabaquismo.

\section{Introdução}

O tabagismo é uma doença de impacto global, considerado um dos principais fatores etiológicos na epidemiologia do câncer e na mortalidade no mundo (West, 2017; Malhotra et al., 2016). O uso do tabaco impacta no aumento do risco de morbidades associadas, em $90 \%$ para neoplasias pulmonares, $85 \%$ para doenças pulmonares obstrutivas crônicas, $30 \%$ para neoplasias de boca, faringe, laringe, esôfago, estômago, pâncreas, rim, bexiga, colo de útero ou mama e $25 \%$ para doenças cerebrovasculares, doença coronariana ou infarto do miocárdio (GBD 2017 Mortality Collaborators, 2018; Centers for Disease Control and Prevention, 2018).

Embora a população geral saiba que o tabaco prejudica a saúde, a maioria dos usuários ainda aceita fumar como parte de suas vidas (Jiang et al., 2019). Uma pesquisa recente de orçamentos familiares no Brasil apontou que há maior gasto com fumo do que com consultas médicas, legumes e verduras, livros didáticos e revistas técnicas ou exames diversos, o que desafia estratégias nutricionais, educativas e assistenciais no âmbito de prevenção e tratamento ao câncer por tabagismo (Instituto Brasileiro de Geografia e Estatística, 2019). A indústria do tabaco atrai o consumo por meio de aditivos aromatizantes (Lisko et al., 2014) e pela multiplicidade de usos (inalado, aspirado ou mascado), mas que não diminuem o risco ou prejuízo à saúde (Barua et al., 2018; Kalkhoran et al., 2018). O câncer bucal pode ser advindo em especial pelo fumo ou ainda uso de tabaco em outras formas (Chaturvedi et al., 2019).

A mucosa bucal ao ser exposta a substâncias do tabaco, como nicotina, dióxido de carbono, formaldeído, hidrocarbonetos aromáticos policíclicos, derivados fenólicos e óxido nítrico, torna-se suscetível a danos celulares devido aos múltiplos metabólitos reativos gerados (Fernández-González \& Oliva, 2018). Reações oxidativas nos tecidos podem causar danos às proteínas, carboidratos, lipídios e DNA, manifestando-se fisicamente em lesões pré-malignas e malignas (Freitas et al., 2016). A boca constitui uma região de fácil acesso e o diagnóstico de lesões em estágio inicial seria facilitado frente a outras regiões anatômicas, por meio de biópsias e exames histopatológicos (Domingos et al., 2014). 
A citologia esfoliativa é um procedimento alternativo para diagnóstico em patologia bucal, que permite a detecção de danos celulares e genotóxicos (Almirón et al., 2015). Aberrações cromossômicas, como micronúcleos (MN), picnose, binucleação ou anucleação (Geus et al., 2019), broken eggs, dentre outras, são biomarcadores de exposição aos agentes prejudiciais do tabaco nos cromossomos (Biswas et al., 2014). Os MN são formações globulares de DNA, compostos por fragmentos cromossômicos ou cromossomos inteiros, não incorporados ao núcleo da célula filha ao final do processo de divisão celular (Degen et al., 2017).

O teste de MN em células da mucosa bucal constitui uma metodologia experimental de fácil aplicabilidade com seres humanos (Ferreira et al., 2021; Fernandes et al., 2020). Entretanto, o diagnóstico de MN por citopatologia é pouco explorado e bastante heterogêneo na comunidade científica, com alta variabilidade geopopulacional de frequências. Para cada 100 células epiteliais em fumantes, Dosi et al. (2016) na Índia observaram 23 MN, Shafi (2015) no Iraque mostrou que uma incidência de 12 MN, enquanto Lazalde-Ramos et al. (2017) no México verificaram em nativos uma frequência de 33 MN.

No intuito de poder contribuir para o rastreamento de genotoxicidade subclínica, ainda sem patologias intraorais detectáveis ao exame clínico, o objetivo deste trabalho visou determinar a frequência de MN nas células epiteliais da mucosa bucal hígida em pacientes fumantes e não fumantes.

\section{Metodologia}

\subsection{Desenho do estudo}

O estudo seguiu um modelo de abordagem quantitativa, descritiva, observacional e transversal (Pereira et al., 2018). O referencial metodológico da pesquisa, para coleta de esfregaços bucais e posterior análise citomorfométrica, foi baseado em Jiménez (2019).

\subsection{Aspectos éticos}

Foram adotados os princípios bioéticos vigentes no Brasil da Resolução do Conselho Nacional de Saúde n. 466/2012 e houve aprovação pelo Comitê de Ética em Pesquisas envolvendo Seres Humanos local (CEP/UVA-Sobral/CE, CAAE n. 50957615.1.0000.5054, protocolo n. 1.403.786). Os pesquisadores realizaram esclarecimento prévio da pesquisa a todos que aceitassem participar voluntariamente, sendo registrada a anuência por meio do Termo de Consentimento Livre e Esclarecido.

\subsection{Cenário e amostra}

Participaram como voluntários os pacientes que buscaram atendimento no serviço odontológico do setor de Diagnóstico Oral da Clínica Integrada da Universidade Federal do Ceará do Campus de Sobral, entre julho de 2016 e junho de 2017. Como critérios de inclusão, foram considerados: faixa etária adulta, entre 40 e 60 anos, com distribuição paritária entre os sexos, histórico de consumo intenso de tabaco ( $\geq 20$ unidades por dia) ou não (controle). Foram excluídos pacientes: com alguma lesão na mucosa bucal detectável depois do exame clínico; com ficha de anamnese, exame clínico e citológico apresentando preenchimento incorreto ou incompleto; sem relato de consumo intenso de tabaco (para os fumantes); ou com consumo adicional de álcool (para os não fumantes).

Para se chegar ao cálculo amostral, foi considerado inicialmente o quantitativo populacional cadastrado no banco de prontuários do setor no mesmo interstício de um ano, perfazendo 34 pacientes elegíveis conforme os critérios de inclusão e exclusão, sendo 10 homens e 24 mulheres. A partir desse universo, foi delineada uma amostragem aleatória randomizada, por meio de sorteio de iguais oportunidades para ambos os sexos. A amostra final foi constituída por 16 pacientes, sendo 8 homens e 8 mulheres, distribuídos em dois grupos: fumantes e não fumantes. 


\subsection{Coleta de dados}

Antes da coleta de células da mucosa bucal, os pacientes selecionados enxaguaram a boca com água corrente para eliminar a presença de restos de alimentos e diminuir a concentração bacteriana. Foi aplicada a técnica de citologia esfoliativa utilizando um cytobrush individual descartável para cada região bucal por paciente: mucosa jugal, borda lateral de língua e assoalho de boca (Figura 1).

Figura 1 - Coleta de amostras intrabucais por citologia esfoliativa.

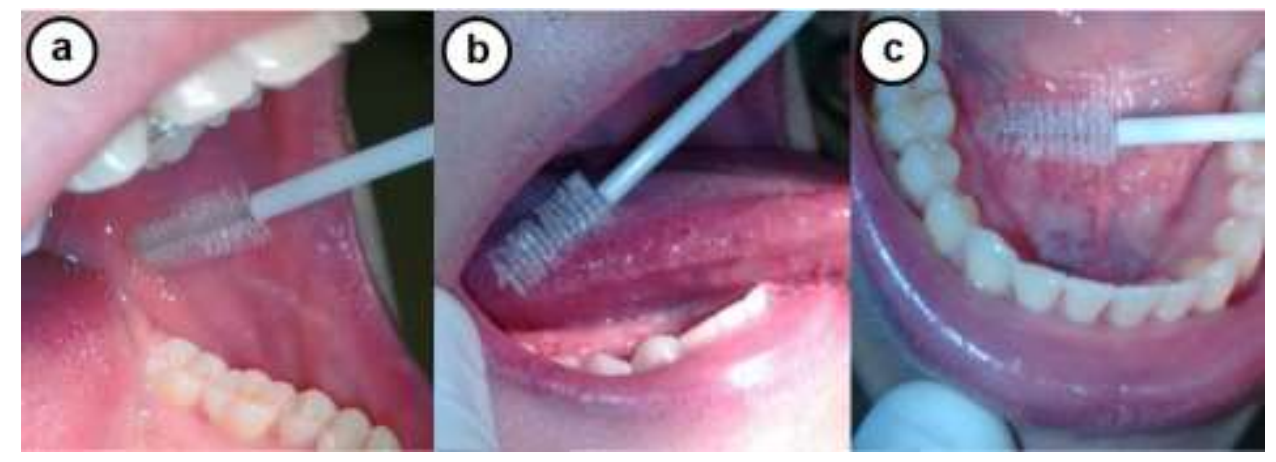

Mucosa jugal (a), borda lateral de língua (b) e assoalho de boca (c). Fonte: Autores (2021).

As 3 lâminas coletadas por paciente foram fixadas em etanol (95\%) por 24 horas, processadas e coradas pela técnica de Papanicolaou (kit Laborclin ${ }^{\circledR}$, Curitiba, PR, Brasil). Nesta técnica citológica, o núcleo cora-se no tom de magenta, resultante da hidrólise ácida da 2-desoxirribose, o açúcar da molécula de DNA. O citoplasma cora-se de rosa avermelhado em células mais superficiais do epitélio oral, com maior concentração de citoqueratina, ou de verde para células intermediárias, com menor queratinização. Esta contra-coloração permitiu uma melhor observação de pequenos fragmentos nucleares, além de minimizar as interferências nas leituras citogenéticas.

\subsection{Análise de dados}

As fotomicrografias foram obtidas com uma câmera digital Cybershot DSC-W300 (Sony, Manaus, Brasil) conectada a um microscópio óptico FWL-1000 (FWL-1000; Feldman Wild Leitz, Manaus, Brasil) com magnificação de 1.000x com auxílio de óleo de imersão. Para cada uma das 48 lâminas histológicas coradas, 100 imagens fotográficas não-superpostas de células individualizadas e bem distendidas foram registradas. A análise citomorfométrica foi realizada por um patologista experiente.

Para as 2.400 células obtidas por grupo ou o total de 4.800 fotomicrografias, foram consideradas: (i) presença de células com micronúcleo (CMN) e (ii) quantidade de micronúcleos por célula (MN/C). Para determinação de MN, foram considerados os critérios citomorfológicos de Tolbert et al. (1992): localização (intracitoplasmática, próxima ao núcleo principal), tamanho (1/16 a 1/3 do diâmetro no núcleo principal), coloração (similar ao núcleo principal, ocasionalmente mais intensa ou mais pálida) e forma (ovalada ou redonda) (Figura 2). 
Figura 2 - Fotomicrografia de queratinócito esfoliado de mucosa jugal normal.

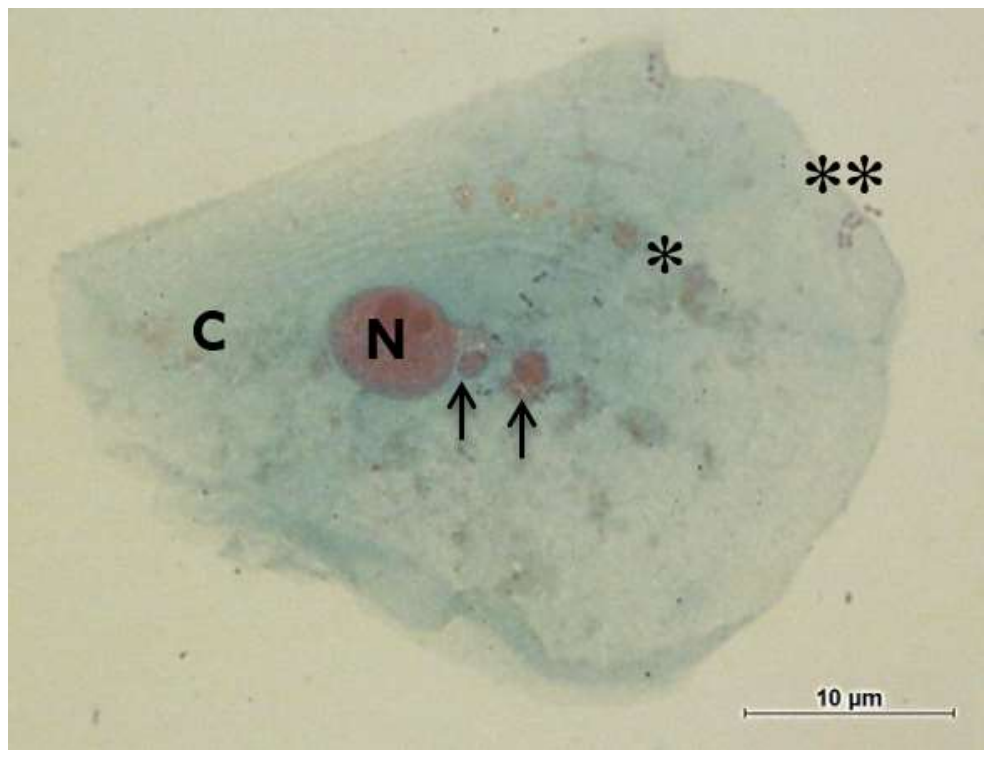

Citoplasma $(\mathrm{C})$, núcleo $(\mathrm{N})$, micronúcleos $(\boldsymbol{\uparrow})$, grânulos de querato-hialina $(*)$ e bactérias $(* *)$. Coloração: Papanicolaou. Magnificação: 1000x. Fonte: Autores (2021)

Foram excluídas da análise outras possíveis alterações citogenéticas (binucleação, picnose, broken egg, cariorrexe ou cariólise), grânulos citoplasmáticos de querato-hialina (de menor tamanho, amorfos e com coloração diferente da nuclear) ou unidades formadoras de colônia bacteriana sobrepostas aos queratinócitos em análise.

Um banco de dados absolutos foi construído no software Excel (Microsoft Office, EUA), de acordo com grupo (fumantes ou não fumantes) e as variáveis independentes (região anatômica, sexo e faixa etária). Para sua interpretação, as análises foram divididas em 2 grandes blocos: (i) presença de CMN/número total de células por região anatômica e (ii) quantidade de MN/C, seguindo as faixas de 1, 2, 3 ou $\geq 4$ micronúcleos.

Os dados paramétricos foram analisados com auxílio do software Prism 7.0 (GraphPad software, CA, EUA) e expressos em média \pm desvio padrão, na forma de gráficos para quantificação de CMN ou tabular para quantificação de MN/C. A análise estatística foi conduzida por análise de variância (ANOVA) e pós-teste de comparações múltiplas de Tukey, considerando diferenças significantes de $\mathrm{p}<0,05$.

Os pacientes que apresentassem maior quantidade de CMN foram informados de sua situação e acompanhados por meio de revisões periódicas, como medida preventiva de potenciais lesões orais clínicas.

\section{Resultados}

A análise morfológica dos queratinócitos esfoliados de mucosa bucal hígida confirmou maior presença de CMN no grupo de fumantes frente ao grupo de não fumantes ( $(p<0,05)$, na razão de 1,95:1 (Figura 3a). Nas regiões bucais isoladas, o assoalho da boca de pacientes fumantes apresentou mais CMN em relação aos não fumantes ( $(<<0,05)$, na razão de 3,64:1, sem diferenças entre as demais regiões (Figura 3b). A respeito da variável sexo, foi possível constatar uma diferença de CMN no sexo feminino entre fumantes e não fumantes ( $\mathrm{p}<0,01)$, na razão de $3,04: 1$, enquanto no masculino não houve diferenças (Figura 3c). Com relação a idade, houve uma diferença de CMN na faixa etária entre 50-60 anos entre fumantes e não fumantes ( $p<0,05$ ), na razão de 2,96:1 (Figura 3d). 
Figura 3 - Média \pm desvio padrão de células micronucleadas (CMN) segundo amostra geral (a), regiões anatômicas intraorais (b), sexo (c) e idade (d).

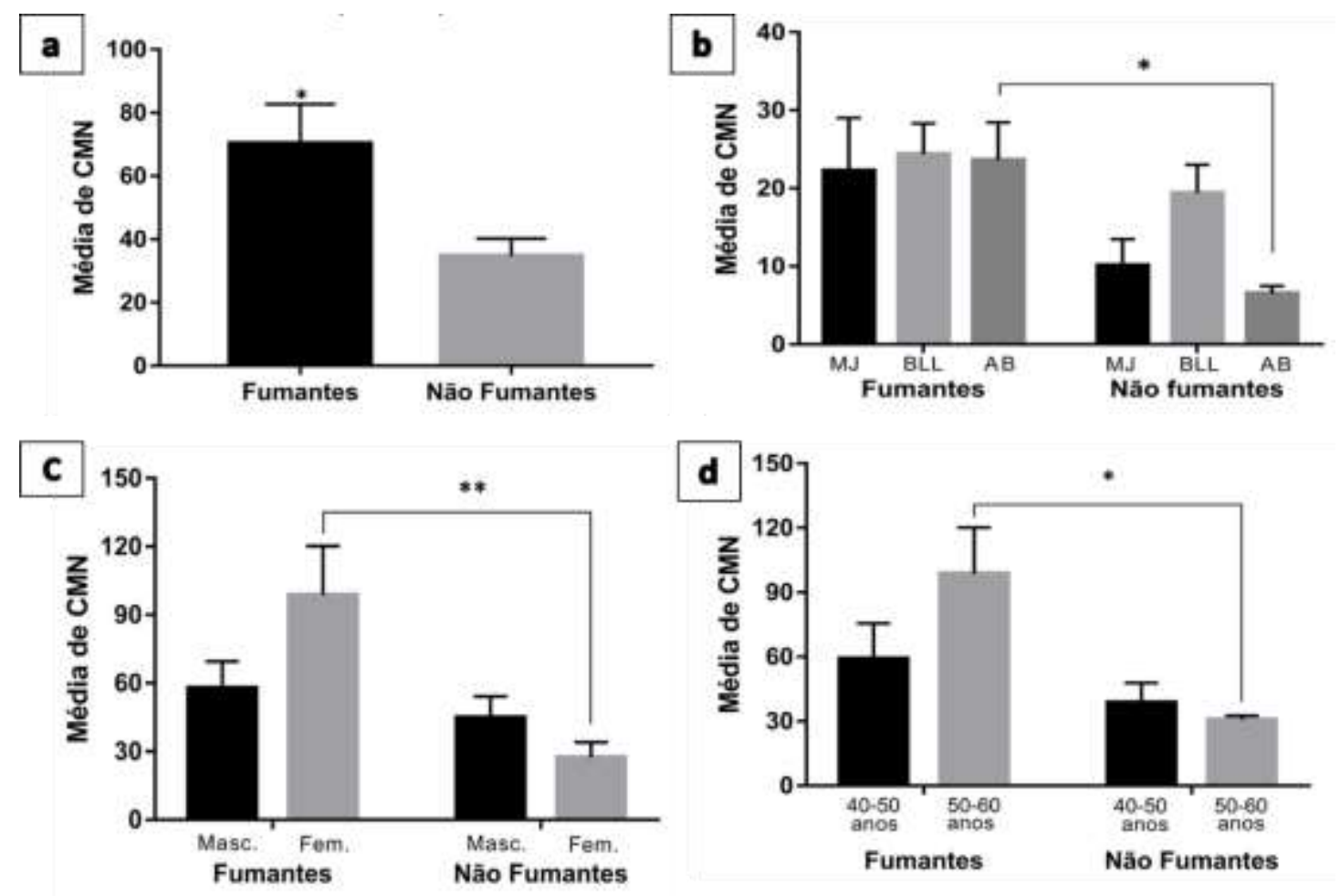

Mucosa jugal (MJ), Borda lateral de língua (BLL) e Assoalho de boca (AB). Análise estatística por teste two-way ANOVA seguido por Tukey, ${ }^{*} \mathrm{p}<0,05, * * \mathrm{p}<0,01$. Fonte: Autores (2021).

A comparação da frequência de MN/C entre pacientes fumantes e não fumantes, segundo a variável região anatômica intraoral, mostrou aumento para as frequências de 1 e $2 \mathrm{MN} / \mathrm{C}$ no grupo fumante frente ao não fumante (p<0,001). Dentro de cada grupo, se observou que as frequências de 3 e $\geq 4 \mathrm{MN} / \mathrm{C}$ foram diferentes, em fumantes ( $<<0,01$ e $p<0,01$, respectivamente) e não fumantes ( $\mathrm{p}<0,001$ e $\mathrm{p}<0,01$, respectivamente). Para mucosa jugal, borda lateral da língua e assoalho da boca, houve diferenças entre fumantes e não fumantes ( $p<0,0001, p<0,01$ e $p<0,0001$, respectivamente). (Tabela 1)

Segundo a variável sexo, observou-se no sexo masculino que no grupo fumantes a quantidade de MN/C variou, ao comparar a frequência de $1 \mathrm{MN} / \mathrm{C}$ com as frequências de 3 e $\geq 4 \mathrm{MN} / \mathrm{C}(\mathrm{p}<0,05)$; também se observou diferença com o grupo não fumantes nas mesmas frequências $(\mathrm{p}<0,05)$. O sexo feminino do grupo fumante apresentou diferença entre a frequência de $1 \mathrm{MN} / \mathrm{C}$ e as frequências de 3 e $\geq 4 \mathrm{MN} / \mathrm{C}$ ( $<<0,01$ e $\mathrm{p}<0,001$, respectivamente), entre a frequência de $1 \mathrm{MN} / \mathrm{C}$ e as frequências de $1 \mathrm{MN} / \mathrm{C}(\mathrm{p}<0,05), 2,3 \mathrm{e} \geq 4 \mathrm{MN} / \mathrm{C}$ entre fumantes e não fumantes $(\mathrm{p}<0,001)$. Dentro do grupo de não fumantes, existiu uma diferença entre o sexo masculino e feminino em relação à frequência de $1 \mathrm{MN} / \mathrm{C}$ e frequências $2,3 \mathrm{e} \geq 4 \mathrm{MN} / \mathrm{C}$ ( $\mathrm{p}<0,05$, $\mathrm{p}<0,01$ e $\mathrm{p}<0,01$ respectivamente). (Tabela 1 )

Para a variável idade, houve na faixa de 50-60 anos uma diferença entre fumantes e não fumantes na frequência de 1 MN/C ( $<<0,001)$. A faixa de 50-60 anos também apresentou diferença entre a frequência de $1 \mathrm{MN} / \mathrm{C}$ e as frequências de 3 e $\geq 4$ MN/C em fumantes ( $p<0,01$ e $p<0,001)$ e não fumantes $(p<0,05$ e $p<0,05)$, respectivamente. Outra diferença ocorreu na faixa etária de 40-50 anos de pacientes não fumantes na frequência de $1 \mathrm{MN} / \mathrm{C}$ e frequências de 3 e $\geq 4 \mathrm{MN} / \mathrm{C}$ (p<0,05). (Tabela 1) 
Tabela 1 - Média \pm desvio padrão da faixa de micronúcleos por célula (MN/C) de pacientes fumantes e não fumantes de acordo com as regiões intraorais, sexo e idade.

\begin{tabular}{|c|c|c|c|c|c|c|}
\hline & Variável & & Faixa & MN/C & & p-valor \\
\hline & & 1 & 2 & 3 & $\geq 4$ & \\
\hline & Fumantes & $31.50 \pm 15,45^{\text {aаa }}$ & $19,88 \pm 11,48^{\text {aаa }}$ & $10,00 \pm 5,15^{b b}$ & $8,88 \pm 5,77^{b b}$ & \\
\hline & Mucosa jugal & $11,25 \pm 10,26$ & $6,63 \pm 5,71$ & $2,25 \pm 2,92$ & $2,13 \pm 2,10$ & aаaа \\
\hline 䨔 & Borda lateral da língua & $10,50 \pm 4,66$ & $7,13 \pm 3,14$ & $3,13 \pm 1,73$ & $3,63 \pm 3,34$ & aа \\
\hline$\stackrel{\Xi}{=}$ & Assoalho da boca & $9,75 \pm 4,92$ & $6,13 \pm 4,91$ & $4,63 \pm 3,20$ & $3,13 \pm 2,64$ & aаaа \\
\hline$\tilde{z}$ & Não fumantes & $22,50 \pm 10,17$ & $9,25 \pm 6,07$ & $2,75 \pm 2,31^{b b b}$ & $1,50 \pm 2,27^{b b}$ & \\
\hline "ా & Mucosa jugal & $5,88 \pm 5,51$ & $2,75 \pm 3,01$ & $1,25 \pm 1,49$ & $0,25 \pm 0,71$ & \\
\hline & Borda lateral da língua & $12,50 \pm 5,40$ & $4,88 \pm 4,52$ & $1,13 \pm 0,99$ & $0,88 \pm 1,73$ & \\
\hline & Assoalho da boca & $4,13 \pm 2,30$ & $1,63 \pm 0,92$ & $0,38 \pm 0,52$ & $0,38 \pm 0,52$ & \\
\hline & Fumantes & $31,50 \pm 15,45$ & $19,88 \pm 11,48$ & $10,00 \pm 5,15$ & $8,88 \pm 5,77$ & \\
\hline & Masculino & $24,25 \pm 6,70$ & $17,00 \pm 7,79$ & $7,75 \pm 2,99^{\mathrm{b}, \mathrm{a}}$ & $8,75 \pm 6,50^{\mathrm{b}, \mathrm{a}}$ & \\
\hline$\dot{x}$ & Feminino & $38,75 \pm 19,28^{a}$ & $22,75 \pm 15,00^{\text {aаa }}$ & $12,25 \pm 6,29^{\mathrm{bb}, \mathrm{aa}}$ & $9,00 \pm 5,94^{\text {bbb,aaa }}$ & \\
\hline w & Não fumantes & $22,50 \pm 10,17$ & $9,25 \pm 6,07$ & $2,75 \pm 2,31$ & $1,50 \pm 2,27$ & \\
\hline & Masculino & $27,50 \pm 11,70$ & $12,25 \pm 6,40$ & $3,00 \pm 2,58$ & $2,00 \pm 2,71$ & \\
\hline & Feminino & $17,50 \pm 6,14$ & $6,25 \pm 4,57^{c}$ & $2,50 \pm 2,38^{\mathrm{cc}}$ & $1,00 \pm 2,00^{\mathrm{cc}}$ & \\
\hline & Fumantes & $31,50 \pm 15,45$ & $19,88 \pm 11,48$ & $10,00 \pm 5,15$ & $8,88 \pm 5,77$ & \\
\hline & $40-50$ anos & $25,00 \pm 8,00$ & $17,00 \pm 9,54$ & $1,00 \pm 0,15$ & $1,13 \pm 0,33$ & \\
\hline$\frac{\mathscr{\nu}}{\tau}$ & $50-60$ anos & $35,40 \pm 18,30^{\text {aаaа }}$ & $21,60 \pm 13,24$ & $11,20 \pm 5,93^{\mathrm{bb}}$ & $8,80 \pm 5,17^{\text {bbb }}$ & \\
\hline & Não fumantes & $22,50 \pm 10,17$ & $9,25 \pm 6,07$ & $2,75 \pm 2,31$ & $1,50 \pm 2,27$ & \\
\hline & $40-50$ anos & $26,25 \pm 13,00$ & $12,50 \pm 6,56$ & $4,50 \pm 1,91^{b}$ & $2,75 \pm 2,75^{b}$ & \\
\hline & $50-60$ anos & $18,75 \pm 5,91$ & $6,00 \pm 3,83$ & $1,00 \pm 0,82^{b}$ & $0,25 \pm 0,50^{b}$ & \\
\hline
\end{tabular}

Análise estatística pelo teste two-way ANOVA seguido por pós-teste de Tukey. Diferenças intergrupos: ${ }^{\mathrm{a}} \mathrm{p}<0,05,{ }^{\mathrm{a} a} \mathrm{p}<0,01,{ }^{\mathrm{aaa}} \mathrm{p}<0,001$, ${ }^{a a a a} \mathrm{p}<0,0001$. Diferenças intragrupos: ${ }^{\mathrm{b}} \mathrm{p}<0,05,{ }^{\mathrm{bb}} \mathrm{p}<0,01,{ }^{\mathrm{bbb}} \mathrm{p}<0,001$. Diferenças entre subgrupos de cada grupo: ${ }^{\mathrm{c}} \mathrm{p}<0,05,{ }^{\mathrm{cc}} \mathrm{p}<0,01$. Fonte: Autores (2021).

\section{Discussão}

A citopatologia é uma potente ferramenta para o diagnóstico laboratorial. No ano de 2018, foram realizados pelo Sistema Único de Saúde 415 exames de citologia oncótica para lábio e cavidade oral, embora a biópsia persista como padrão ouro, com um total de 20.797 procedimentos anuais realizados (Instituto Nacional do Câncer, 2020). Neste contexto, o teste de micronúcleos em células esfoliadas da mucosa oral é uma boa fonte para biomonitoramento de danos genéticos, por ser técnica viável, não invasiva e econômica para avaliar o dano ao DNA (Yadav \& Saini, 2015).

No presente estudo, a frequência geral de CMN da mucosa bucal de fumantes foi marcadamente superior à de não fumantes. Esses resultados são consistentes com a literatura, onde o aumento do número de micronúcleos se deve ao tabagismo (Geus et al., 2019; Degen et al., 2017; Thomas et al., 2017; Dosi et al., 2016; Biswas et al., 2014).

$\mathrm{Na}$ análise da frequência de $\mathrm{CMN}$ nos grupos de fumantes e não fumantes segundo as regiões anatômicas, a maior prevalência em assoalho da boca corrobora achados de Basulto et al. (2019), que reforçam se tratar de uma das regiões mais delicadas da boca e região frequente de ocorrência de neoplasias malignas. Monteiro-Amado et al. (2013) ainda reforçam que a carcinogênese no assoalho de boca pode exibir rápida progressão no sistema TNM e, em consequência, um prognóstico mais 
sombrio. Em contrapartida, Mohanta et al. (2014) argumentam que é a mucosa jugal que possui a maior presença na quantidade de micronúcleos, associada a fatores como estilo de vida, destoando dos nossos resultados.

Com relação à frequência de $\mathrm{CMN}$ em grupos de fumantes e não fumantes, a maior prevalência em mulheres neste estudo confirma a controvérsia existente na literatura, onde há relatos de valores mais altos dessas aberrações cromossômicas em mulheres (Nefic et al., 2013), em homens (Navya et al., 2017) ou ausência de diferenças entre sexos (Mohanta et al., 2014). A ocorrência de novos casos de neoplasia maligna da cavidade oral em cada 100 mil habitantes é maior para mulheres no estado do Ceará se comparada ao panorama nacional (5,05 contra 4,21, respectivamente), diferente de homens (Ceará: 6,82 e Brasil: 11,27), bem como é maior o número total de biópsias e de citologia oncótica realizadas em mulheres para diagnóstico de câncer de cavidade oral e lábio no Brasil (Instituto Nacional do Câncer, 2020). Tais evidências reforçam a importância do presente estudo, que serve de alerta para a população feminina exposta a fator de risco para carcinogênese oral.

Em relação à frequência de CMN em grupos de fumantes e não fumantes, de acordo com a idade, nesta investigação não houve maior expressividade na faixa etária de 40 a 50 anos; no entanto, outras investigações observaram diferença significativa nesta faixa etária (Ferraz et al., 2016; Yadav \& Saini, 2015). A faixa etária de 50-60 anos, com diferença significativa entre os grupos estudados, destoa dos resultados encontrados por Nefic et al. (2013), onde não houve diferenças entre homens e mulheres segundo a maior idade, apesar de ser hipotetizado que marcadores citogenéticos fossem mais abundantes. Os efeitos da senilidade poderiam ser explicados como uma combinação de processos geneticamente programados e alterações genéticas induzidas por fatores endógenos e exógenos, como o tabaco (Ferraz et al., 2016).

Alguns estudos traçam uma convergência direta entre presença de CMN ou MN/C com lesões pré-malignas ou câncer de boca (Geus et al., 2019; Jiang et al., 2019; Biswas et al., 2014). Entretanto, cabe ressaltar que o presente estudo apresenta como limitação não inferir uma relação prognóstica determinística entre a expressividade de micronúcleos e a ocorrência de uma lesão clínica, o que só poderia ser analisado com maior espectro amostral e acompanhamento a longo prazo.

Todos os pacientes que participaram deste estudo receberam aconselhamento antitabágico e são acompanhados a longo prazo, em conformidade aos protocolos internacionais (Barua et al., 2018). Estratégias diagnósticas e educativas se revestem de ainda maior relevância diante do atual cenário da pandemia por Covid-19, onde tabagismo constitui alto fator de risco que aumenta a morbi-mortalidade da doença e precisa ser encarado com seriedade, para aumento da qualidade e expectativa de vida (Castro-Silva \& Maciel, 2020; Leonel et al., 2020; Maciel et al., 2020).

\section{Conclusão}

Este trabalho evidenciou diferenças na quantidade de células micronucleadas e quantidade de micronúcleos por célula de mucosa bucal, de acordo com hábito tabágico, região anatômica intraoral, sexo e faixa etária. Tais achados contribuem para valorizar a citopatologia como método diagnóstico subclínico de alterações genotóxicas celulares mediadas pelo tabaco, bem como a importância do rastreamento epidemiológico em população exposta a fator de risco para carcinogênese oral.

Os presentes resultados fomentam futuros estudos longitudinais em lesões pré-malignas ou neoplasias epiteliais malignas de cavidade bucal diagnosticadas clinicamente, onde seria possível elucidar o valor prognóstico de micronúcleos e da citopatologia na prática odontológica.

\section{Agradecimentos}

Ao Governo do Estado do Ceará, à Fundação Cearense de Apoio ao Desenvolvimento Científico e Tecnológico (FUNCAP, processo n. 88881.166822/2018-01/Edital 04/2017), à Coordenação de Aperfeiçoamento de Pessoal de Nível Superior (CAPES, bolsas do Programa de Demanda Social, código 001), ao Conselho Nacional de Desenvolvimento Científico e Tecnológico (CNPq, bolsa de Iniciação Científica) e à Universidade Federal do Ceará (UFC, Programa de Apoio à Pós- 
graduação/PROAP e Programa Institucional de Bolsas de Iniciação Científica/PIBIC), pelo apoio financeiro. A toda a equipe de trabalho do Laboratório de Biomateriais do Programa de Pós-graduação em Biotecnologia (PPGB-UFC Sobral), Curso de Odontologia (UFC Sobral) e Grupo de Estudos em Morfologia (GEM-UFC Sobral), pelo apoio na concepção, coleta e análise dos dados e escrita desta pesquisa.

\section{Referências}

Almirón, M. S., Montenegro, M. A., Gili, M. A. \& Segovia, S. M. (2015). Importancia de la citología exfoliativa en el diagnóstico de lesiones bucales. Revista de la Facultad de Odontología, 8 (1), 26-33. http://dx.doi.org/10.30972/rfo.811628.

Barua, R. S., Rigotti, N. A., Benowitz, N. L., Cummings, K. M., Jazayeri, M. A., Morris, P. B., Ratchford, E. V., Sarna, L., Stecker, E. C. \& Wiggins, B. S. (2018). 2018 ACC expert consensus decision pathway on tobacco cessation treatment. Journal of the American College Cardiology, 72 (25), $3332-3365$. https://www.jacc.org/doi/pdf/10.1016/j.jacc.2018.10.027.

Basulto, R. M., Rodríguez, Y. M. R. \& Beltrán, J. V. (2019). Estudio clínico-epidemiológico de pacientes con cáncer en suelo de boca. MediSur, 17 (5), 698705. http://scielo.sld.cu/pdf/ms/v17n5/1727-897X-ms-17-05-698.pdf.

Biswas, S. D., Garai, B. \& Banerjee, M. (2014). Genotoxic effects of tobacco on buccal epithelium: Cell Nuclear Anomalies as Biomarker. Asia Pacific Journal of Medical Toxicology, 3 (4), 157-162. http://dx.doi.org/10.22038/APJMT.2014.3843.

Castro-Silva, I. I. \& Maciel, J. A. C. (2020). Panorama de pesquisas com seres humanos sobre covid19 no Brasil. Revista Bioética, 28 (4), 655-663. https://doi.org/10.1590/1983-80422020284429.

Centers for Disease Control and Prevention. (2018). Smoking \& Tobacco Use: Health Effects of Cigarette Smoking. https://www.cdc.gov/tobacco/data_statistics/fact_sheets/health_effects/effects_cig_smoking/index.htm.

Chaturvedi, P., Singh, A., Chien, C.-Y. \& Warnakulasuriya, S. (2019). Tobacco related oral cancer. BMJ, 365 (2142), 1-10. https://doi.org/10.1136/bmj.12142.

Degen, A. N., Valiatti, T. B., Freitas, V. M., Azambuja-de-Souza, D. P., Romão, N. F. \& Sobral, F. O. S. (2017). Avaliação de micronúcleos em células da mucosa de fumantes e não fumante. UNILUS Ensino $e \quad$ Pesquisa, 14 (36), http://revista.unilus.edu.br/index.php/ruep/article/view/871/u2017v14n36e871.

Domingos, P. A. S., Passalacqua, M. L. C. \& Martins-de-Oliveira, A. L. B. (2014). Câncer bucal: um problema de saúde pública. Revista de Odontologia da Universidade Cidade de São Paulo, 26 (1): 46-52. http://publicacoes.unicid.edu.br/index.php/revistadaodontologia/article/view/285/182.

Dosi, T., Gupta, D., Hazari, A., Rajput, R., Chauhan, P. \& Rajapuri, A. S. (2016). Assessment of micronuclei frequency in individuals with a habit of tobacco by means of exfoliated oral buccal cells. Journal of International Society Preventive Community Dentistry, 6 (Suppl 2), S143-147. https://www.ncbi.nlm.nih.gov/pmc/articles/PMC5022392/.

Fernandes, C. L. F., Brum, R. L. \& Schwantes, L. (2020). O ensaio de micronúcleo como uma ferramenta de ensino nas escolas. Research, Society and Development, 9 (4), e102942892. http://dx.doi.org/10.33448/rsd-v9i4.2892.

Fernández-González, E. M. \& Oliva, D. A. F. (2018). Tabaquismo y su relación con las enfermedades cardiovasculares. Revista Habanera de Ciencias Médicas, 17 (2), 225-235. http://scielo.sld.cu/scielo.php?script=sci_arttext\&pid=S1729-519X2018000200008.

Ferraz, G. A., Costa, A. O., Cerqueira, E. M. M. \& Meireles, J. R. C. (2016). Efeitos da idade sobre as frequências de micronúcleos e alterações nucleares degenerativas. Revista Brasileira de Geriatria e Gerontologia, 19 (4), 627-634. https://doi.org/10.1590/1809-98232016019.150155.

Ferreira, E. C. C., Gonçalves, T. M., Pereira, T. M., Hasna, A. A., Oliveira, F. E., Jorjão, A. L., Camargo, S. E. A., Oliveira, L. D., Spalding, M. (2021). Research, Society and Development, 10 (1), e37610111902. http://dx.doi.org/10.33448/rsd-v10i1.11902.

Freitas, R. M., Rodrigues, A. M. X., Matos Júnior, A. F. \& Oliveira, G. A. L. (2016). Fatores de risco e principais alterações citopatológicas do câncer bucal: uma revisão de literatura. Revista Brasileira de Análises Clínicas, 48 (1), 13-18. http://www.rbac.org.br/artigos/fatores-de-risco-e-principais-alteracoescitopatologicas-do-cancer-bucal-uma-revisao-de-literatura/.

GBD 2017 Mortality Collaborators. (2018). Global, regional, and national age-sex-specific mortality and life expectancy, 1950-2017: a systematic analysis for the Global Burden of Disease Study 2017. The Lancet, 392 (10159), 1684-1735. https://doi.org/10.1016/S0140-6736(18)31891-9.

Geus, J. L., Wambier, L. M., Loguercio, A. D. \& Reis, A. (2019). The smokeless tobacco habit and DNA damage: a systematic review and meta-analysis. Medicina Oral Patología Oral y Cirurgía Bucal, 24 (2), 145-155. https://doi.org/10.4317/medoral.22846.

Instituto Brasileiro de Geografia e Estatística. (2019). Pesquisa de Orçamentos Familiares 2017-2018. https://biblioteca.ibge.gov.br/visualizacao/livros/liv101670.pdf.

Instituto Nacional do Câncer. (2020). Relatório sobre o cenário assistencial e epidemiológico do câncer de lábio e cavidade oral no Brasil. https://www.inca.gov.br/sites/ufu.sti.inca.local/files//media/document/relatorio_cancer_de_boca_2020_0.pdf.

Jiang, X., Wu, J., Wang, J. \& Huang, R. (2019). Tobacco and oral squamous cell carcinoma: A review of carcinogenic pathways. Tobacco Induced Diseases, 17 (29), 1-9. https://doi.org/10.18332/tid/105844.

Jiménez, H. G. Q. (2019). Avaliação subclínica da frequência de micronúcleos em células da mucosa bucal em pacientes fumantes e não fumantes. Sobral: UFC. http://www.repositorio.ufc.br/ri/handle/riufc/50593. 
Kalkhoran, S., Benowitz, N. L. \& Nancy, A. R. (2018). Prevention and treatment of tobacco use: JACC health promotion series. Journal of the American College Cardiology, 72 (9), 1030-1045. https://doi.org/10.1016/j.jacc.2018.06.036.

Lazalde-Ramos, B. P., Zamora-Pérez, A. L., Sosa-Macías, M., Galaviz-Hernández, C. \& Zúñiga-González, G. M. (2017). Micronuclei and nuclear anomalies in Mexico's indigenous population. Salud Pública de México, 59 (5), 532-539. https://doi.org/10.21149/8318.

Leonel, A. C. L. S., Martelli-Junior, H., Bonan, P. R. F., Kowalski, L. P. \& Perez, D. E. C. (2020). COVID-19, head and neck cancer, and the need of training of health students and practitioners regarding to tobacco control and patient counseling. Oral Oncology, 106, e104739. https://doi.org/10.1016/j.oraloncology.2020.104739.

Lisko, J. G., Stanfill, S. B. \& Watson, C. H. (2014). Quantitation of ten flavor compounds in unburned tobacco products. Analytical Methods, 6 (13), 46984704. http://dx.doi.org/10.1039/C4AY00271G.

Maciel, J. A. C., Castro-Silva, I. I. \& Farias, M. R. (2020). Initial analysis of the spatial correlation between the incidence of COVID-19 and human development in the municipalities of the state of Ceará in Brazil. Revista Brasileira de Epidemiologia, 23, e200057. https://doi.org/10.1590/1980549720200057

Malhotra, J., Malvezzi, M., Negri, E., La Vecchia, C. \& Boffetta, P. (2016). Risk factors for lung cancer worldwide. European Respiratory Journal, 48 (3), 889-902. http://dx.doi.org/10.1183/13993003.00359-2016.

Mohanta, A., Mohanty, P. K. \& Parida, G. (2014). Keratinized tadpole cells in human oral neoplasm: a cytodiagnostic approach. IOSR Journal of Dental and Medical Sciences, 3 (9), 110-119. http://www.iosrjournals.org/iosr-jdms/papers/Vol13-issue9/Version-6/U01396110119.pdf.

Monteiro-Amado, F., Castro-Silva, I. I., Lima, C. J., Soares, F. A., Kowalski, L. P. \& Granjeiro, J. M. (2013). Immunohistochemical evaluation of MMP-2, MMP-9 and CD31/microvascular density in squamous cell carcinomas of the floor of the mouth. Brazilian Dental Journal, 24 (1), 3-9. https://doi.org/10.1590/0103-6440201301941.

Navya, B. N., Najem, H. \& Alva, S. R. (2017). Comparison of cytogenetic abnormality of exfoliative buccal cells among smokers and non-smokers. Archives of Cytology and Histopathology Research, 2 (1), 1-4. https://www.achr.co.in/article-details/3895.

Nefic, H., Musanovic, J., Kurteshi, K., Prutina, E. \& Turcalo, E. (2013). The effects of sex, age and cigarette smoking on micronucleus and degenerative nuclear alteration frequencies in human buccal cells of healthy Bosnian subjects. Journal of Health Sciences, 3 (3), 196-204. https://core.ac.uk/download/pdf/73974398.pdf.

Pereira, A. S., Shitsuka, D. M., Parreira, F. J. \& Shitsika, R. (2018). Metodologia da pesquisa científica. UFSM. https://repositorio.ufsm.br/bitstream/han dle/1/15824/Lic_Computacao_Metodologia-Pesquisa-Cientifica.pdf?sequence=1.

Shafi, F. A. A. (2015). The effects of smoking on micronucleus frequencies in buccal cells of healthy iraqi individuals. World Journal of Phamaceutical Research, 4 (9), 406-415. https://wjpr.net/admin/assets/article_issue/1441020097.pdf.

Thomas, T. M., Ramesh, M. \& Seka, B. (2017). Study of exfoliated cells in the buccal mucosa of smokers and non-smokers - a cytological comparative study. International Journal of Current Advanced Research, 6 (8), 5010-5014. http://dx.doi.org/10.24327/ijcar.2017.5014.0633.

Tolbert, P. E., Shy, C. M. \& Allen, J. W. (1992). Micronuclei and other nuclear anomalies in buccal smears: methods development. Mutation Research, 271 (1), 69-77. https://doi.org/10.1016/0165-1161(92)90033-i.

West, R. (2017). Tobacco smoking: Health impact, prevalence, correlates and interventions. Psychology \& Health, 32 (8), $1018-1036$. https://doi.org/10.1080/08870446.2017.1325890.

Yadav, A. S. \& Saini, M. (2015). Increased frequency of nuclear anomalies in exfoliated buccal mucosa of cigarette smokers. Journal of Entomology and Zoology Studies, 3 (2), 7-10. https://www.entomoljournal.com/archives/?year=2015\&vol=3\&issue=2\&ArticleId=148. 